\title{
Scaling Up Safer Birth Bundle Through Quality Improvement in Nepal (SUSTAIN) - a stepped wedge cluster randomized controlled trial in public hospitals
}

Rejina Gurung ${ }^{1}$, Anjani Kumar Jha², Susheel Pyakurel ${ }^{3}$, Abhishek Gurung ${ }^{1}$, Helena Litorp ${ }^{4}$, Johan Wrammert ${ }^{4}$, Bijay Kumar Jha ${ }^{2}$, Prajwal Paudel ${ }^{5}$, Syed Moshfiqur Rahman ${ }^{4}$, Honey Malla', Srijana Sharma ${ }^{1}$, Manish Gautam ${ }^{5}$, Jorgen Erland Linde ${ }^{6}$, Md Moinuddin $^{7}$, Uwe Ewald ${ }^{4}$, Mats Målqvist ${ }^{4}$, Anna Axelin ${ }^{8}$ and Ashish $\mathrm{KC}^{4,9^{*}}$ (D)

\begin{abstract}
Background: Each year, 2.2 million intrapartum-related deaths (intrapartum stillbirths and first day neonatal deaths) occur worldwide with $99 \%$ of them taking place in low- and middle-income countries. Despite the accelerated increase in the proportion of deliveries taking place in health facilities in these settings, the stillborn and neonatal mortality rates have not reduced proportionately. Poor quality of care in health facilities is attributed to two-thirds of these deaths. Improving quality of care during the intrapartum period needs investments in evidence-based interventions. We aim to evaluate the quality improvement package—Scaling Up Safer Bundle Through Quality Improvement in Nepal (SUSTAIN) —on intrapartum care and intrapartum-related mortality in public hospitals of Nepal.
\end{abstract}

Methods: We will conduct a stepped wedge cluster randomized controlled trial in eight public hospitals with each having least 3000 deliveries a year. Each hospital will represent a cluster with an intervention transition period of 2 months in each. With a level of significance of $95 \%$, the statistical power of $90 \%$ and an intra-cluster correlation of 0.00015 , a study period of 19 months should detect at least a 15\% change in intrapartum-related mortality. Quality improvement training, mentoring, systematic feedback, and a continuous improvement cycle will be instituted based on bottleneck analyses in each hospital. All concerned health workers will be trained on standard basic neonatal resuscitation and essential newborn care. Portable fetal heart monitors (Moyo ${ }^{\oplus}$ ) and neonatal heart rate monitors (Neobeat ${ }^{\oplus}$ ) will be introduced in the hospitals to identify fetal distress during labor and to improve neonatal resuscitation. Independent research teams will collect data in each hospital on intervention inputs, processes, and outcomes by reviewing records and carrying out observations and interviews. The dose-response effect will be evaluated through process evaluations.

(Continued on next page)

\footnotetext{
* Correspondence: aaashis7@yahoo.com

${ }^{4}$ Department of Women's and Children's Health, Uppsala University, Dag Hammarskjölds väg 14B, Uppsala, Sweden

${ }^{9}$ Society of Public Health Physicians Nepal, Lalitpur, Nepal

Full list of author information is available at the end of the article
}

(c) The Author(s). 2019 Open Access This article is distributed under the terms of the Creative Commons Attribution 4.0 International License (http://creativecommons.org/licenses/by/4.0/), which permits unrestricted use, distribution, and reproduction in any medium, provided you give appropriate credit to the original author(s) and the source, provide a link to the Creative Commons license, and indicate if changes were made. The Creative Commons Public Domain Dedication waiver (http://creativecommons.org/publicdomain/zero/1.0/) applies to the data made available in this article, unless otherwise stated. 
(Continued from previous page)

Discussion: With the global momentum to improve quality of intrapartum care, better understanding of QI package within a health facility context is important. The proposed package is based on experiences from a similar previous scale-up trial carried out in Nepal. The proposed evaluation will provide evidence on QI package and technology for implementation and scale up in similar settings.

Trial registration number: ISRCTN16741720. Registered on 2 March 2019.

Keywords: Quality improvement interventions, Basic neonatal resuscitation, Fetal heart rate monitoring, Stepped wedge cluster randomized control trial, Nepal

\section{Contributions to the literature}

- Quality improvement packages improve perinatal care in public hospitals.

- New technologies for fetal heart rate monitoring and neonatal heart rate monitoring tend to be well accepted by health care providers.

- To evaluate the adequacy of the implementation of a QI package (the Scaling Up Birth Bundle Through Quality Improvement in Nepal [SUSTAIN] package) in public hospitals using a Stepped Wedge Cluster Randomized Controlled Trial (SW-CRCT)

- The effect of the Ql package on health worker's adherence to intrapartum care (fetal heart rate monitoring and neonatal resuscitation) as per standard protocol and on intrapartum survival.

\section{Background}

The accelerated reduction in maternal and child mortality during the Millennium Development Goal era (20002015) led to the realization that further reduction can only be achieved with improved quality of care in the intrapartum period $[1,2]$. Every year, almost 1.2 million stillbirths and 250,000 maternal deaths occur during the intrapartum period, and a million newborns die in their first day of life [3-5]. The United Nations' Every Woman and Every Child strategy 2016-2030 aims to reduce preventable maternal, neonatal, and child deaths by the end of the Sustainable Development period 2030 [6]. The strategy has resulted in a number of efforts to identify ways of reducing preventable deaths [7]. One of the key initiatives was led by the Lancet Global Health Commission for High Quality Health Systems in the SDG Era [8]. Through consultations and systematic reviews, the commission produced a framework for improved quality of care in health care settings $[9,10]$. Its five foundations are (1) understanding populations' health care needs; (2) strengthening structures and governance for improving quality of care at all levels of health systems [11]; (3) redesigning and optimizing the health workforce to provide a more conducive environment for health care provision; (4) introducing new tools, quality improvement interventions, and technologies for delivering health care; and (5) the adequacy or capacity of health facilities to deliver health care as per the demands of their client populations [8].

The PARiHS framework (Promoting Action on Research Implementation in Health Services) promotes the translation of evidence into practice using context and facilitation as interplay for improving quality of care $[12,13]$. This is a useful framework as two-thirds of premature and preventable neonatal death are due to poor quality health care and not to lack of access to health care [14]. The large investments made in improving access to and the availability of maternal and newborn care since the start of the MDG period in 2001 [15] had a large impact on reducing the extent of the first and second delays of maternal and newborn care [16]. However, the third delay of inadequate quality of care remains a large challenge $[17,18]$.

In Nepal, several studies have found inadequate quality of care as a major barrier to reducing maternal and neonatal deaths, especially during the intrapartum period $[19,20]$. The following approaches for quality improvement have been effective in low-income settings to overcome this barrier [21-23]:

- Understanding the context in which health facilities operate, and based on this improving leadership to bring about organizational improvements [24];

- Facilitating quality improvement through external and internal drivers such as mentoring and feedback from audits [25] for bringing improvements in clinical units [26].

- Introducing new standards and tools for improving efficiency to implement the standards.

- Setting up data platforms for instigating accountability for the provision of quality care [27].

The actual 'dose-response' of the above four approaches will depend upon the adequacy of QI package implementation and the context of implementation [28].

The implementation research will examine the impact of a new quality improvement project--the Scaling Up 
Birth Bundle Through Quality Improvement in Nepal (SUSTAIN). The SUSTAIN project aims to improve intrapartum care through a set of quality improvement interventions (Fig. 1). The interventions are based on learning from our previous implementation research on quality improvement for intrapartum care [29].

The objective of the research is to evaluate:

- The impact of the SUSTAIN package on intrapartum-related mortality;

- The impact of the package on health workers' performance on monitoring fetal heart rates, essential newborn care, and neonatal resuscitation;

- The appropriateness of implementing the quality improvement interventions in the hospitals [30]; and

- The acceptability of the SUSTAIN package in the hospitals.

- The perception of women for intrapartum care

\section{Methods}

\section{Trial design}

The trial is a stepped wedge cluster randomized controlled trial in eight public hospitals in Nepal. Each hospital will represent a cluster randomized in a cross-sectional wedge design with intervention transition period in each hospital in 2 months (Fig. 2). With the qualitative research components, we aim to provide insight into women's and staffs' response to intervention and to understand how contextual factor affect its implementation [31].

\section{Settings}

Each hospital manages at least 3000 deliveries a year (Table 1). Their intrapartum-related mortality rates range from 11 to 36.5 intrapartum-related deaths (intrapartum stillbirth and first-day mortality) per 1000 births. All the hospitals have separate labor units and operating theaters. Normal and complicated vaginal deliveries take place in the labor units and cesarean sections in the operating theaters. Each hospital has a postnatal unit and a sick newborn care unit. The hospitals provide level II sick newborn care services [32].

\section{Participants}

Eligible criteria for participants The study will cover pregnant women with a gestational age of more than 22 weeks admitted to hospital with fetal heart sound at admission who consent to be enrolled in the study. The process evaluation data are collected from staff members working in the labor and delivery room in the study sites.

\section{Interventions}

The SUSTAIN package is a bundled QI package to empower health care workers to provide improved care and to review its provision during the intrapartum and immediate postpartum periods. The interventions include training and technology for intrapartum monitoring, neonatal resuscitation, and a supporting system to review the implementation of these measures.

The SUSTAIN interventions in the hospitals will be as follows:

1. The bottleneck analysis of delivery care and setting up mechanisms to review and plan improved care and improve accountability.

2. The introduction of the Safer Births Bundle, which is a set of tools for training and therapy to improve

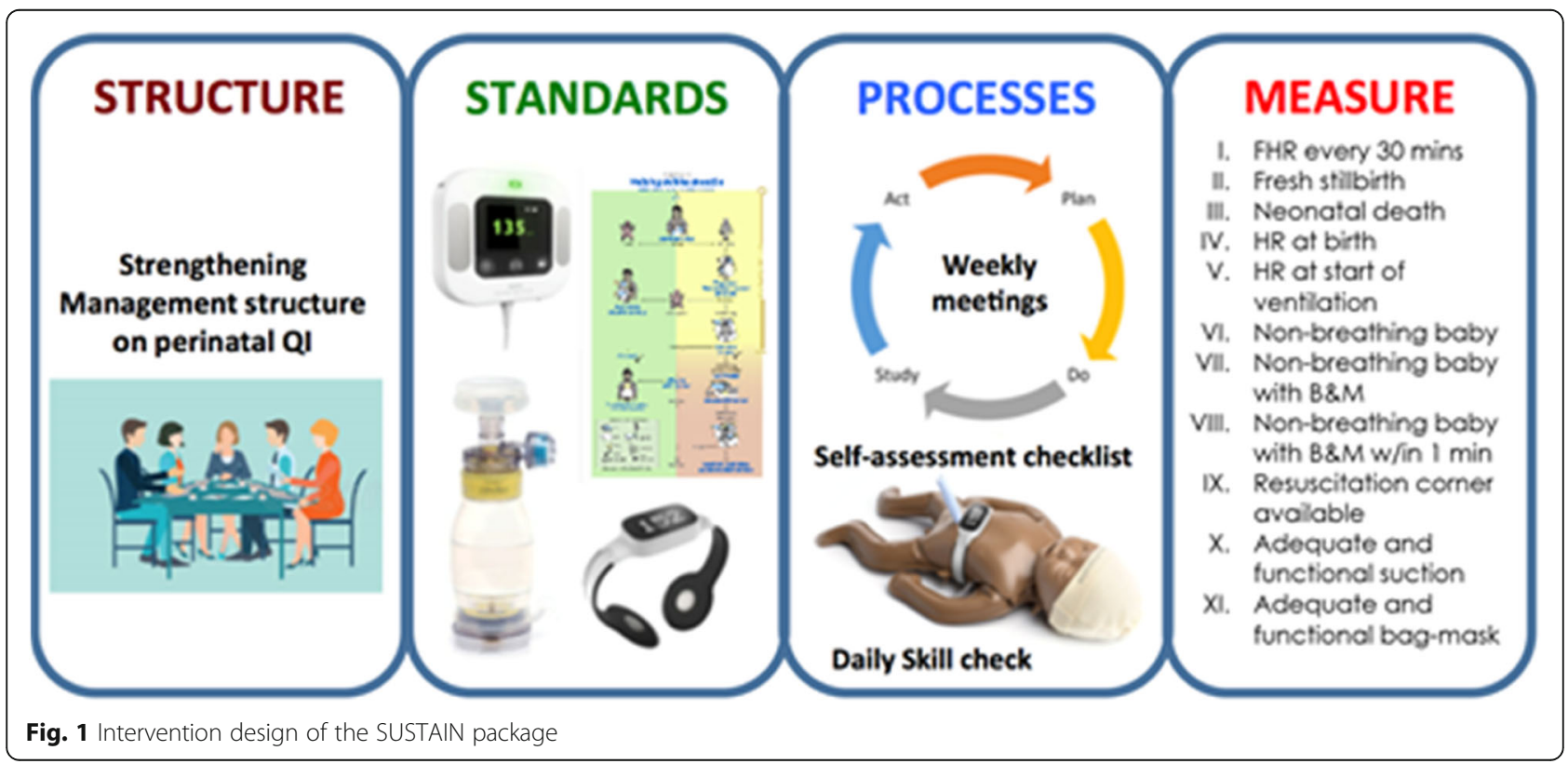




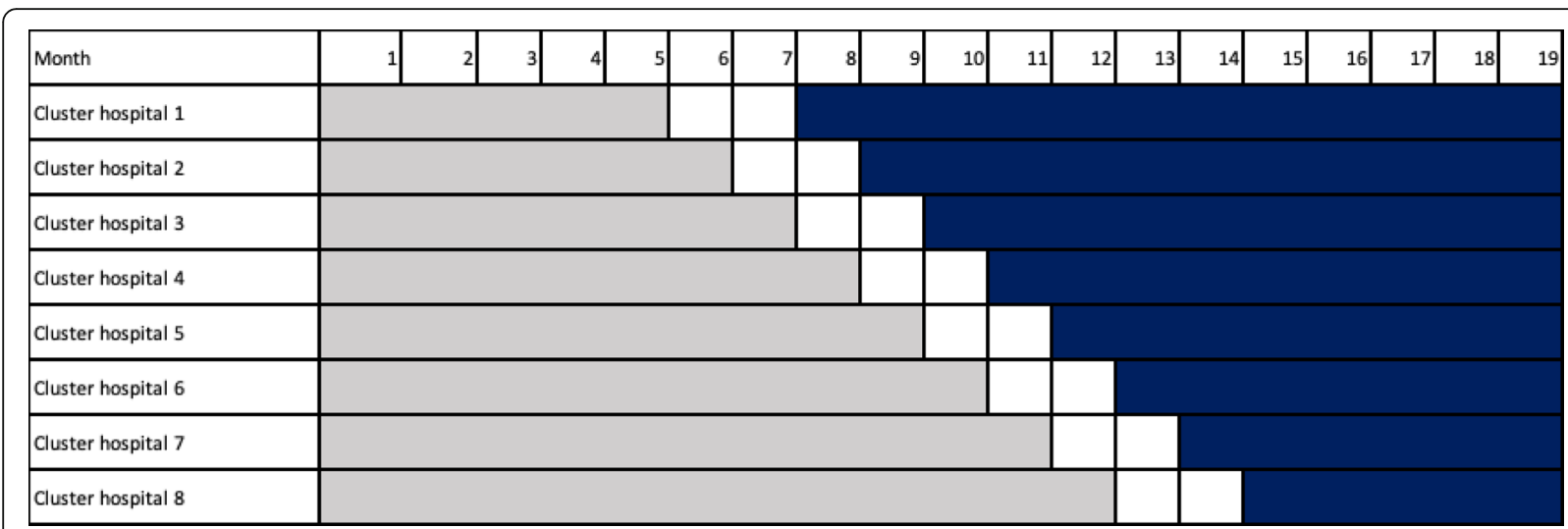

Note: Grey shading is the control period, white is the implementation transition period and dark blue is the intervention period.

Fig. 2 Stepped wedge cluster randomized controlled design. Note: gray shading is the control period, white is the implementation transition period, and dark blue is the intervention period

the monitoring of labor (using Moyo FHR monitors ${ }^{\circ}$ ) [33] and neonatal resuscitation (using upright bag-masks ${ }^{\circ}$ [34], NeoBeat ${ }^{\circ}$ newborn heart rate meters and NeoNatalie live training manikins ${ }^{\circ}$ ).

3. The implementation of QI interventions in delivery rooms including daily skill checks on neonatal resuscitation, using checklists to prepare for births and resuscitation, using self-review evaluation checklists after neonatal resuscitation incidents, and holding weekly review meetings to track progress on implementing the new tools and standards.

4. The setting up of a system to continuously measure the quality of intrapartum care using the Plan-DoStudy-Act (PDSA) approach [35]. This approach harnesses the local ownership of challenges and provides an actionable framework to monitor and evaluate progress to improve and sustain QI changes.

In the project using the i-PARISH framework, facilitation is done at three different levels of types of facilitators. First, an external facilitator or mentor who introduces the innovation of SUSTAIN to the hospital leadership and conducts an assessment of the hospital context in terms of implementing the innovation. The external facilitator will be an expert to coordinate with the different networks of facilitators. The external facilitators conduct bottleneck analysis workshop, plan meeting, and supervise the internal facilitators. Second, an experienced internal facilitator has a deeper understanding of the organization local context. The facilitator conducts training on the intervention package and the rolls out of the innovations (safer births bundle). Third, an internal new facilitator will be working under the supervision of the experienced internal facilitator to implement the daily skill checks on neonatal resuscitation, using checklists to prepare for births and resuscitation, using self-review evaluation checklists after neonatal resuscitation incidents, and conducting PDSA meeting.

\section{Outcomes}

Primary outcome

- Intrapartum-related mortality-intrapartum-related mortality defined as intrapartum stillbirth (no breathing

Table 1 Total birth and mortality rate in the study hospitals (2017)

\begin{tabular}{lllll}
\hline & Total no. births & $\begin{array}{l}\text { Intrapartum stillbirth rate } \\
\text { (per 1000 births) }\end{array}$ & $\begin{array}{l}\text { First-day neonatal mortality rate } \\
\text { (per 1000 live births) }\end{array}$ & $\begin{array}{l}\text { Intrapartum-related death rate } \\
\text { (per 1000 births) }\end{array}$ \\
\hline Koshi Zonal Hospital & 5464 & 9.3 & 4.4 & 13.7 \\
Janakpur Regional Hospital & 14,300 & 11.9 & 23 & 34.9 \\
Bharatpur Hospital & 11,006 & 7.3 & 3.7 & 11.0 \\
Lumbini Zonal Hospital & 8649 & 11.2 & 12.3 & 23.5 \\
Dadeldhura Sub-Regional Hospital & 3112 & 10.3 & 4.4 & 14.7 \\
Mid-Western Regional Hospital & 3847 & 5.0 & 25 & 30.0 \\
Bheri Zonal Hospital & 4132 & 1.2 & 35.3 & 36.5 \\
Seti Zonal Hospital & 6277 & 2.2 & 29.7 & 27.9
\end{tabular}

Intrapartu-related mortality is the composite of intrapartum stillbirths and first-day mortality data 
10 min after delivery) and neonatal death within the first $24 \mathrm{~h}$ of life [36].

Secondary outcomes:

- Proportion of deliveries with fetal heart rate monitoring as per standard protocol.

- Proportion of deliveries in which abnormal fetal heart rate during labor is followed by neonatal resuscitation.

- Proportion of deliveries resulting in emergency cesarean sections and instrumental deliveries due to fetal distress.

- Proportion of non-breathing babies who receive a bag and mask ventilation within 1 min of birth.

- Proportion of health workers maintaining neonatal resuscitation skills 6 months after being trained on it.

- Adequacy and acceptability of the implementation of SUSTAIN package

\section{Sample size}

The intervention will be employed in the same hospital. Therefore, every hospital will be included in control as well as intervention groups. This fact will produce the correlated data between the arms. For binary correlated data, we have considered the one-sided McNemar's test for sample size calculation. Considering the statistical power of $90 \%$, level of significance at $5 \%$ and the average cluster size of 4800 deliveries we have calculated the sample size. For adjusting the cluster effect, the sample size is adjusted by the design effect. The design effect is chosen as a proxy of 1.71 based on Nepal's Demographic Health Survey 2016 [37]. This design effect is updated based on our average cluster size. The design effect for NMR in NDHS 2016 is reported as 1.12 for urban sample with an average cluster size of 799. Based on the information, we have calculated the intra-cluster correlation coefficient (ICC) of 0.00015 which gives the design effect 1.71 for average cluster size 4800 [38]. We have also considered the 7\% loss to follow up to reach the final sample size of 31,259 deliveries in each group. This number will be proportionally allocated among the hospitals. The STATA command "power" is used for necessary calculation.

Purposive sampling will be used in the qualitative components of the study. Maximum variation sampling will guide the selection of participants to in-depth interviews with health workers and focus group discussions with the hospital management team. This approach secures a wide variety of people of interest and consequently a broad range of perspectives to better understand contextual factors influencing on implementation. A convenient sample of pregnant women will be interviewed about their experiences of intrapartum care. Sample sizes will be based on data saturation (Table 2).

\section{Randomization}

Using a simple random technique, the principal investigator will generate a random sequence among the eight hospitals to determine the temporal stepped wedge sequence of the clusters through simple random technique. The allocation of the introduction of intervention in each hospital will be done in a stepped wedge pattern based on the above randomly generated sequence. Blinding is part of the SW-RCT-clusters/hospitals will not know when the other hospitals will be controls or intervention.

\section{Data collection}

An independent team will be established in each hospital to collect data on implementation, process, and outcomes.

Implementation level data-a form will be used to collect data on the number of stakeholders engaged in each intervention:

- A hospital readiness and service availability assessment tool will be used to conduct the bottleneck analysis of service readiness for intrapartum care. A planning tool will be used to develop a plan based on the bottleneck analysis.

- Data on health workers' knowledge and skills on intrapartum care before and after training will be collected using multiple-choice questions and skills checklist.

- The data on the continuous quality improvement process will be collected using a Plan-Do-Study-Act diary, which was tested in our previous study [21].

- The periodic performance of the health workers will be done using a skills checklist.

Process level data-A separate system will be established to collect data on health worker performance in simulated and clinical intrapartum care settings:

- Data will be collected on the use of the NeoNatalie Live ${ }^{\bullet}$ manikin through skill drills in an application-based system.

- The use of fetal heart rate monitor $\left(\right.$ Neobeat $\left.^{\circ}\right)$ will be assessed by a separate group of data collector using an observation checklist

- Data on health worker performance on monitoring fetal heart rates, immediate newborn care, and neonatal resuscitation will be collected using an observation checklist.

- The implementation of the SUSTAIN interventions using the PARIHS framework [39] will be evaluated through in-depth interviews and focus group discussions with service providers and caregivers.

- The acceptability of the new interventions will be assessed by studying health worker acceptance and barriers to use. 
Table 2 Number of health workers in the study hospitals (2017)

\begin{tabular}{|c|c|c|c|c|}
\hline & Nurses & Doctors & Other health workers & Total health workers \\
\hline Koshi Zonal Hospital & 25 & 8 & 45 & 78 \\
\hline Janakpur Regional Hospital & 60 & 22 & 0 & 82 \\
\hline Bharatpur Hospital & 86 & 20 & 17 & 123 \\
\hline Lumbini Zonal Hospital & 81 & 7 & 36 & 124 \\
\hline Dadeldhura Sub-Regional Hospital & 42 & 2 & 13 & 57 \\
\hline Mid-Western Regional Hospital & 20 & 3 & 22 & 45 \\
\hline Bheri Zonal Hospital & 33 & 4 & 26 & 63 \\
\hline Seti Zonal Hospital & 32 & 6 & 56 & 94 \\
\hline
\end{tabular}

- The perceptions of women about intrapartum care will be evaluated with semi-structured interviews.

Outcome level data-data on mortality and clinical events during intrapartum care will be collected from patient case notes and labor and delivery registers.

Socio-demographic data-the socio-demographic characteristics of the women will be collected through semistructured interviews.

Equipment data-signal data and events that are automatically recorded by the Safer Births equipment will be uploaded to a cloud service with strict access control. Equipment data will be analyzed to complement the process level data and drive local QI processes, they will be analyzed to check the integrity and condition of the equipment and the data will be used to create new insight, develop improved equipment as well new signal analysis methods.

\section{Data management}

The study will maintain the confidentiality of individual participants including their identity and location. To protect against data loss, all data will be collected in a tablet-based application and kept on a secure server.

In each hospital, a data collection coordinator will assess the quality and completeness of the data. The data collected on a paper-based format from the hospitals will be indexed and a master ID will be provided to each data entry form. Prior to data entry, the completed forms will be reviewed for missing variable(s) and the open-ended responses will be coded. The data will be entered into a CS-Pro database.

\section{Data analysis}

A data cleaning and data analysis strategy will be developed once the data has been collected. The implementation and process level data will be evaluated using a Medical Research Council process evaluation process [40]. The outcome level data will be analyzed using the CONSORT guidelines for processing quantitative data [41] and COREQ for processing the qualitative data [42].
For the analysis i-PARIHS framework will be used, which essentially involves what is to be implemented, who with, where, and how. The assessment of the evidence of innovation in the SUSTAIN package, characteristics of the different stakeholders to whom the interventions were targeted, characteristics of the settings, and implementation of the facilitation process. The facilitation implemented through a different level of facilitators (external, internal experienced, and new) using different quality improvement interventions to improve the structure, standards, and process will be assessed.

Inductive thematic network analysis will be used to analyze interview data [43]. The audio recorded interviews are transcribed, and verbatim data will be transferred to NVivo Version 12.1.0. The initial coding will be done by research assistants. In the next phase, senior researchers will familiarize themselves with data and critically reviewed codes. Analyses will continue with collating codes in several basic themes, creating organizing themes from basic themes, and developing global theme by combining all data.

\section{Ethics}

The study protocol has been finalized through a consultative process with professional bodies, academia, global experts, and Nepal's Ministry of Health and Population. Ethical approval has been received from the Ethical Review Board of the Nepal Health Research Council. Written consent will be taken from all study participants. A data safety monitoring board will be formed to monitor the progress of the research and any deviations from the protocol [44]. The researchers will strive to carry out the study to the highest level of integrity in line with the World Medical Association Declaration of Helsinki Ethical Principles for Medical Research [45].

\section{Discussion}

We will study the impact of an evidence-based package of QI interventions and technologies for improving the quality of intrapartum care and hope that the research will provide useful evidence on a scalable model of QI 
Table 3 Intensity of each QI item in the SUSTAIN project

\begin{tabular}{lll}
\hline Quality improvement package items & Details & Number \\
\hline Facilitators training on HBS & Training on continuous quality improvement package. Checklist use HBS package & 6 days \\
& $\begin{array}{l}\text { An orientation with hospital leadership on the improvement of care process for mothers } \\
\text { and newborn } \\
\text { Orientation to facilitators on the tools for bottleneck analysis }\end{array}$ & $2 \mathrm{~h}$ \\
& Assessment of maternal and newborn services in the hospital & $2-4 \mathrm{~h}$ \\
& Bottleneck analysis of maternal and newborn services & 2 days \\
& Development of problem-solving process using the PDSA approach & Half day \\
Training & Training health workers on the HBS package and continuous quality improvement (CQI) & 3 days \\
Mentoring & Monthly visit by clinical mentors to guide the implementation of the standards & 1 day each month \\
Audit and feedback & Use of progress board Daily drills & Daily
\end{tabular}

interventions and technologies for improving intrapartum care. The research will provide new information on what context and intensity of facilitation are required to implement the interventions in hospitals in a lowincome setting. The research will evaluate the i-PARISH theoretical framework on the different facilitation strategy through different facilitators to the hospital stakeholders. Furthermore, we aim to construct an evidencebased framework for QI and technology-based solutions to improve intrapartum care. Three tools will be used to translate the evidence into action once the evidence on the process and outcome evaluations are available. First, a plain language summary of the results and on the importance of the QI package will be disseminated to general audiences via the local and national Nepalese media. Second, a brief will be developed for policy-makers outlining the required QI framework for improving care to advocate for more investments in such interventions. Third, the results will be published in a peer-reviewed journal for international academic, researcher, and program experts (Table 3 ).

\section{Abbreviations \\ CONSORT: Consolidated Standards of Reporting Trials; COREQ: Consolidated Criteria for Reporting Qualitative Research; CS: Pro-Census and Survey Processing System; HBS: Helping babies survive; MDG: Millennium Development Goal; PARIHS: Promoting Action on Research Implementation in Health Services; PDSA: Plan-Do-Study-aAct; QI: quality improvement; SDG: Sustainable Development Goal; SUSTAIN: Scaling Up Birth Bundle Through Quality Improvement in Nepal}

\section{Acknowledgements}

We like to acknowledge Dr. Avinash K Sunny, Omkar Basnet, Pratiksya Bhattarai, Asmita Paudel, and Sandhya Baral for the cognitive testing of the observation tools. We want to thank Bhula C. Rai, Sudip Karki, and D. B Gurung for the grant application. We want to thank Steve Keeling for language editing.

\section{Authors' contributions}

AKC, JW, HL, SMR, AG, and RG conceptualized the study. AKC produced the first draft of this protocol. RG and PP made the ethics application to the Nepal Health Research Council. All co-authors reviewed the first draft of the protocol and provided comments on the protocol and study design. All authors have read and approved the final version of the manuscript.

\section{Funding}

This research will be funded by the Canadian Grand Challenges Fund and the Laerdal Foundation for Acute Medicine.

\section{Availability of data and materials}

Not applicable.

\section{Ethics approval and consent to participate}

Ethical approval for the research was received from the Ethical Review Board of the Nepal Health Research Council (ref 110-2019) on 15 February 2019.

Written consent will be taken from all research participants.

\section{Consent for publication}

Not applicable.

\section{Competing interests}

Neither Laerdal nor the funder (Canadian Grand Challenges Fund and the Laerdal Foundation) has influenced the design of the study protocol.

\section{Author details}

'Golden Community, Jwagal, Lalitpur, Nepal. ${ }^{2}$ Ministry of Health and Population, Government of Nepal, Kathmandu, Nepal. ${ }^{3}$ Nepal Health Research Council, Ramshah Path, Kathmandu, Nepal. ${ }^{4}$ Department of Women's and Children's Health, Uppsala University, Dag Hammarskjölds väg 14B, Uppsala, Sweden. ${ }^{5}$ Anweshan, Lalitpur, Nepal. ${ }^{6}$ Department of Paediatrics, Stavanger University Hospital, Våland burrough, Stavanger, Norway. ${ }^{7}$ Maternal and Child Health Division, ICDDR,B, Dhaka, Bangladesh. ${ }^{8}$ University of Turku, Turku, Finland. ${ }^{9}$ Society of Public Health Physicians Nepal, Lalitpur, Nepal.

Received: 5 May 2019 Accepted: 10 June 2019

Published online: 19 June 2019

\section{References}

1. Lawn JE, Blencowe H, Oza S, You D, Lee AC, Waiswa P, Lalli M, Bhutta Z, Barros AJ, Christian P, et al. Every newborn: progress, priorities, and potential beyond survival. Lancet. 2014;384(9938):189-205.

2. Dickson KE, Simen-Kapeu A, Kinney MV, Huicho L, Vesel L, Lackritz E, de Graft Johnson J, von Xylander S, Rafique N, Sylla M, et al. Every newborn: health-systems bottlenecks and strategies to accelerate scale-up in countries. Lancet. 2014;384(9941):438-54.

3. Collaborators GBDCM. Global, regional, national, and selected subnational levels of stillbirths, neonatal, infant, and under-5 mortality, 1980-2015: a systematic analysis for the global burden of disease study 2015. Lancet. 2016;388(10053):1725-74.

4. Collaborators GBDMM. Global, regional, and national levels of maternal mortality, 1990-2015: a systematic analysis for the Global Burden of Disease Study 2015. Lancet. 2016;388(10053):1775-812

5. Oza S, Cousens SN, Lawn JE. Estimation of daily risk of neonatal death, including the day of birth, in 186 countries in 2013: a vital-registration and modelling-based study. Lancet Glob Health. 2014;2(11):e635-44. 
6. Zeid S, Bustreo F, Barakat MT, Maurer P, Gilmore K. For every woman, every child, everywhere: a universal agenda for the health of women, children, and adolescents. Lancet. 2015;385(9981):1919-20.

7. Desalegn H, Solberg E, Kim JY. The global financing facility: country investments for every woman, adolescent, and child. Lancet. 2015;386(9989):105-6.

8. Kruk ME, Gage AD, Arsenault $C$, Jordan K, Leslie HH, Roder-DeWan S, Adeyi O, Barker P, Daelmans B, Doubova SV, et al. High-quality health systems in the sustainable development goals era: time for a revolution. Lancet Glob Health. 2018;6(11):e1196-252.

9. Pantoja T, Opiyo N, Lewin S, Paulsen E, Ciapponi A, Wiysonge CS, Herrera CA, Rada G, Penaloza B, Dudley L, et al. Implementation strategies for health systems in low-income countries: an overview of systematic reviews. Cochrane Database Syst Rev. 2017;9:CD011086.

10. Rowe AK, Rowe SY, Peters DH, Holloway KA, Chalker J, Ross-Degnan D. Effectiveness of strategies to improve health-care provider practices in low-income and middle-income countries: a systematic review. Lancet Glob Health. 2018:6(11):e1163-75.

11. Herrera CA, Lewin S, Paulsen E, Ciapponi A, Opiyo N, Pantoja T, Rada G, Wiysonge CS, Bastias G, Garcia Marti S, et al. Governance arrangements for health systems in low-income countries: an overview of systematic reviews. Cochrane Database Syst Rev. 2017:9:CD011085.

12. Kitson AL, Rycroft-Malone J, Harvey G, McCormack B, Seers K, Titchen A. Evaluating the successful implementation of evidence into practice using the PARiHS framework: theoretical and practical challenges. Implement Sci. 2008;3:1.

13. Rycroft-Malone J. The PARIHS framework-a framework for guiding the implementation of evidence-based practice. J Nurs Care Qual. 2004;19(4):297-304.

14. Kruk ME, Gage AD, Joseph NT, Danaei G, Garcia-Saiso S, Salomon JA. Mortality due to low-quality health systems in the universal health coverage era: a systematic analysis of amenable deaths in 137 countries. Lancet. 2018;392(10160):2203-12.

15. Pitt C, Grollman C, Martinez-Alvarez M, Arregoces L, Borghi J. Tracking aid for global health goals: a systematic comparison of four approaches applied to reproductive, maternal, newborn, and child health. Lancet Glob Health. 2018;6(8):e859-74.

16. Countdown to C. Countdown to 2030: tracking progress towards universal coverage for reproductive, maternal, newborn, and child health. Lancet. 2018:391(10129):1538-48.

17. Arsenault C, Jordan K, Lee D, Dinsa G, Manzi F, Marchant T, Kruk ME. Equity in antenatal care quality: an analysis of 91 national household surveys. Lancet Glob Health. 2018;6(11):e1186-95.

18. Sharma J, Leslie HH, Regan M, Nambiar D, Kruk ME. Can India's primary care facilities deliver? A cross-sectional assessment of the Indian public health system's capacity for basic delivery and newborn services. BMJ Open. 2018;8(6):e020532.

19. Lindback C, Kc A, Wrammert J, Vitrakoti R, Ewald U, Malqvist M. Poor adherence to neonatal resuscitation guidelines exposed; an observational study using camera surveillance at a tertiary hospital in Nepal. BMC Pediatr. 2014;14:233.

20. Joshi C, Torvaldsen S, Hodgson R, Hayen A. Factors associated with the use and quality of antenatal care in Nepal: a population-based study using the demographic and health survey data. BMC Pregnancy Childbirth. 2014;14:94.

21. KC A, Wrammert J, Clark RB, Ewald U, Vitrakoti R, Chaudhary P, Pun A, Raaijmakers $\mathrm{H}$, Malqvist M. Reducing perinatal mortality in Nepal using Helping Babies Breathe. Pediatrics. 2016;137(6).

22. Larson E, Gage AD, Mbaruku GM, Mbatia R, Haneuse S, Kruk ME. Effect of a maternal and newborn health system quality improvement project on the use of facilities for childbirth: a cluster-randomized study in rural Tanzania. Trop Med Int Health. 2019;24(5):636-46.

23. Semrau KEA, Hirschhorn LR, Marx Delaney M, Singh VP, Saurastri R, Sharma N, Tuller DE, Firestone R, Lipsitz S, Dhingra-Kumar N, et al. Outcomes of a coaching-based WHO safe childbirth checklist program in India. N Engl J Med. 2017;377(24):2313-24

24. Helfrich CD, Li YF, Sharp ND, Sales AE. Organizational readiness to change assessment (ORCA): development of an instrument based on the Promoting Action on Research in Health Services (PARIHS) framework. Implement Sci. 2009;4:38.

25. Ivers N, Jamtvedt G, Flottorp S, Young JM, Odgaard-Jensen J, French SD, O'Brien MA, Johansen M, Grimshaw J, Oxman AD. Audit and feedback: effects on professional practice and healthcare outcomes. Cochrane Database Syst Rev. 2012;(6):CD000259.
26. Harvey G, Kitson A. PARIHS revisited: from heuristic to integrated framework for the successful implementation of knowledge into practice. Implement Sci. 2016;11:33.

27. Seers K, Rycroft-Malone J, Cox K, Crichton N, Edwards RT, Eldh AC, Estabrooks CA, Harvey G, Hawkes C, Jones C, et al. Facilitating implementation of research evidence (FIRE): an international cluster randomised controlled trial to evaluate two models of facilitation informed by the Promoting Action on Research Implementation in Health Services (PARIHS) framework. Implement Sci. 2018;13(1):137.

28. Rycroft-Malone J, Seers K, Chandler J, Hawkes CA, Crichton N, Allen C, Bullock I, Strunin L. The role of evidence, context, and facilitation in an implementation trial: implications for the development of the PARIHS framework. Implement Sci. 2013;8:28.

29. Kc A, Bergstrom A, Chaulagain D, Brunell O, Ewald U, Gurung A, Eriksson L, Litorp H, Wrammert J, Gronqvist E, et al. Scaling up quality improvement intervention for perinatal care in Nepal (NePeriQIP); study protocol of a cluster randomised trial. BMJ Glob Health. 2017;2(3):e000497.

30. Proctor E, Silmere H, Raghavan R, Hovmand P, Aarons G, Bunger A, Griffey R, Hensley M. Outcomes for implementation research: conceptual distinctions, measurement challenges, and research agenda. Admin Pol Ment Health. 2011;38(2):65-76.

31. Schumacher KL, Koresawa S, West C, Dodd M, Paul SM, Tripathy D, Koo P, Miaskowski C. Qualitative research contribution to a randomized clinical trial. Res Nurs Health. 2005;28(3):268-80.

32. Stark AR. American Academy of Pediatrics Committee on F, newborn: levels of neonatal care. Pediatrics. 2004:114(5):1341-7.

33. Kamala BA, Ersdal HL, Dalen I, Abeid MS, Ngarina MM, Perlman JM, Kidanto HL. Implementation of a novel continuous fetal Doppler (Moyo) improves quality of intrapartum fetal heart rate monitoring in a resource-limited tertiary hospital in Tanzania: an observational study. PLoS One. 2018;13(10):e0205698.

34. Rafferty AR, Johnson L, Davis PG, Dawson JA, Thio M, Owen LS. Neonatal mannequin comparison of the upright self-inflating bag and snap-fit mask versus standard resuscitators and masks: leak, applied load and tidal volumes. Arch Dis Child Fetal Neonatal Ed. 2018;103(6):F562-6.

35. Seward N, Neuman M, Colbourn T, Osrin D, Lewycka S, Azad K, Costello A Das S, Fottrell E, Kuddus A, et al. Effects of women's groups practising participatory learning and action on preventive and care-seeking behaviours to reduce neonatal mortality: a meta-analysis of clusterrandomised trials. PLoS Med. 2017;14(12):e1002467.

36. Lawn JE, Lee AC, Kinney M, Sibley L, Carlo WA, Paul VK, Pattinson R, Darmstadt GL. Two million intrapartum-related stillbirths and neonatal deaths: where, why, and what can be done? Int J Gynaecol Obstet. 2009;107(Suppl 1):S5-18 S19.

37. Ghimire PR, Agho KE, Renzaho AMN, Nisha MK, Dibley M, Raynes-Greenow C. Factors associated with perinatal mortality in Nepal: evidence from Nepal demographic and health survey 2001-2016. BMC Pregnancy Childbirth. 2019;19(1):88.

38. Pagel C, Prost A, Lewycka S, Das S, Colbourn T, Mahapatra R, Azad K, Costello A, Osrin D. Intracluster correlation coefficients and coefficients of variation for perinatal outcomes from five cluster-randomised controlled trials in low and middle-income countries: results and methodological implications. Trials. 2011;12:151.

39. Bergstrom A, Peterson S, Namusoko S, Waiswa P, Wallin L. Knowledge translation in Uganda: a qualitative study of Ugandan midwives' and managers' perceived relevance of the sub-elements of the context cornerstone in the PARIHS framework. Implement Sci. 2012;7:117.

40. Moore GF, Audrey S, Barker M, Bond L, Bonell C, Hardeman W, Moore L, O'Cathain A, Tinati T, Wight D, et al. Process evaluation of complex interventions: Medical Research Council guidance. BMJ. 2015;350:h1258

41. Hemming K, Taljaard M, McKenzie JE, Hooper R, Copas A, Thompson JA, Dixon-Woods M, Aldcroft A, Doussau A, Grayling M, et al. Reporting of stepped wedge cluster randomised trials: extension of the CONSORT 2010 statement with explanation and elaboration. BMJ. 2018:363:k1614

42. Tong A, Sainsbury P, Craig J. Consolidated criteria for reporting qualitative research (COREQ): a 32-item checklist for interviews and focus groups. Int J Qual Health Care. 2007;19(6):349-57.

43. Attride-Stirling J. Thematic networks: an analytic tool for qualitative research Qual Res. 2001;1(3):385-405. 
44. Damocles Study Group NHSHTAP. A proposed charter for clinical trial data monitoring committees: helping them to do their job well. Lancet. 2005;365(9460):711-22.

45. World Medical A. World Medical association declaration of Helsinki: ethical principles for medical research involving human subjects. JAMA. 2013;310(20):2191-4.

\section{Publisher's Note}

Springer Nature remains neutral with regard to jurisdictional claims in published maps and institutional affiliations.

Ready to submit your research? Choose BMC and benefit from:

- fast, convenient online submission

- thorough peer review by experienced researchers in your field

- rapid publication on acceptance

- support for research data, including large and complex data types

- gold Open Access which fosters wider collaboration and increased citations

- maximum visibility for your research: over $100 \mathrm{M}$ website views per year

At BMC, research is always in progress.

Learn more biomedcentral.com/submissions 